\title{
CONOCIMIENTOS, ACTITUDES Y PRÁCTICAS ACERCA DE PARASITOSIS INTESTINAL EN ADULTOS QUE ASISTIERON A CONSULTA DE ATENCIÓN PRIMARIA EN LA COMUNIDAD DE PAYA MUNICIPIO BANÍ, PROVINCIA PERAVIA, REPÚBLICA DOMINICANA DURANTE EL PERIODO MAYO-JUNIO 2018
}

\author{
Knowledge, behavior and practices regarding intestinal parasitism in \\ adults who received primary care examination in the community of Paya, \\ municipality of Baní, province Peravia, Dominican Republic, during the \\ period May-June 2018
}

\author{
Sarah Nidia Mercedes Peralta Menaa, Tommy Paul Guerrero Pimentel ${ }^{\mathbf{b}}$ y Emiltón López
}

Recibido: 6 de abril, 2019 • Aprobado: 14 de julio, 2019

Cómo citar: Mercedes Peralta Mena SN, Guerrero Pimentel TP, López E. Conocimientos, actitudes y prácticas acerca de parasitosis intestinal en adultos que asistieron a consulta de atención primaria en la comunidad de Paya municipio Baní, provincia Peravia, República Dominicana durante el perí́odo mayo-junio 2018. cysa [Internet]. 22 de noviembre de 2019 [citado 25 de noviembre de 2019];3(3):69-8. Disponible en: https://revistas.intec.edu.do/index.php/cisa/article/view/1536

\section{Resumen}

Introducción: el parasitismo intestinal es un problema de gran prevalencia a nivel de salud pública mundial, responsable de aproximadamente el $10 \%$ de las diarreas en humanos. Las parasitosis intestinales son infestaciones producidas por parásitos cuyo hábitat natural es el aparato digestivo de las personas y animales. Es una de las principales causas de consulta en las clínicas rurales. También, es una de las más importantes causas de malnutriciones en poblaciones pediátricas.

Material y métodos: este estudio fue de carácter prospectivo, descriptivo y de corte transversal. Para adquirir la información discutida se empleó un protocolo (ver sección de anexos) basado en una investigación previa y adaptada por los autores. Se tomaron en cuenta las fichas familiares de los pacientes.

\footnotetext{
a Estudiantes de Medicina del Instituto Tecnológico de Santo Domingo (INTEC). Correo-e: sarahnidiaperalta@gmail.com

b Estudiantes de Medicina del Instituto Tecnológico de Santo Domingo (INTEC). Correo-e: tommyguerrero@gmail.com
}

\begin{abstract}
Introduction: Intestinal parasitism represents a very prevalent public health care issue worldwide, being responsible for approximately $10 \%$ of cases of diarrhea in humans. Intestinal parasitoses are infestations produced by parasites whose natural habitat is the digestive system of people and animals. It is one of the main motives of consult in rural clinics. Also, it's one of the most important causes of malnutrition in pediatric populations.
\end{abstract}

Materials and Methods: This study was of prospective, descriptive and transversal nature. Information was gathered through the use of a questionnaire (see Annexes) based upon a previous research and it was adapted by the authors.

The studied population was all the patients from Paya Arriba, which have assisted to check-ups in the Primary Care Unit of Paya. The inclusion criteria required the

\footnotetext{
c Doctor en Medicina, Coordinador Internado Social del Instituto Tecnológico de Santo Domingo (INTEC).

Correo-e: emiltonlopez@hotmail.com
} 
La población estudiada corresponde a todos los pacientes de Paya Arriba que asistieron a la consulta en la Unidad de Atención Primaria de Paya. Como criterio de inclusión se requirió que la persona fuese mayor de edad, y que se identificase a sí mismo como cabecera del hogar, residente permanente de la comunidad de Paya Arriba y que voluntariamente participara en el estudio.

Conclusión: el $60 \%$ de los entrevistados está consciente de que tiene un nivel intermedio de conocimientos, ya que se autoevaluaron a sí mismos con un tres de cinco, siendo el cinco el mayor puntaje. Mientras que un $28 \%$ se autoevaluó con dos de cinco, lo que pone de manifiesto que está consciente de la necesidad de educación en el tema.

Se exhorta a los habitantes a recalcar lo imprescindible de acciones tan sencillas como el lavado de manos para promover salud, para promover vida.

Palabras clave: parasitosis; conocimiento; actitud; práctica; determinantes de salud.

\section{Introducción}

La Organización Mundial de la Salud estima que más de 2 billones de personas en el mundo viven con enfermedades debido a los parásitos intestinales, especialmente en países en desarrollo ${ }^{1}$. La OPS/ OMS calcula que 20-30 \% de todos los latinoamericanos están infectados por helmintos intestinales (parásitos intestinales), mientras que las cifras en los barrios pobres alcanzan con frecuencia el $50 \%$ y hasta el $95 \%$ en algunos grupos indígenas ${ }^{2}$.

El parasitismo intestinal es un problema de gran prevalencia a nivel de salud pública mundial, responsable de aproximadamente el $10 \%$ de las diarreas en humanos. Las parasitosis intestinales son infestaciones producidas por parásitos cuyo hábitat natural es el aparato digestivo de las personas y animales. Tienen distribución mundial, aunque están estrechamente ligadas a la pobreza y a las malas condiciones higiénico-sanitarias, por lo que aparecen más frecuentemente en países en vías de desarrollo ${ }^{3}$.

Es una de las principales causas de consulta en las clínicas rurales, junto a las infecciones de vías person to be of age, to identify him/her as the family's head, to be a permanent resident of the community of Paya Arriba and to agree voluntarily to participate in the study.

Conclusion: Around $60 \%$ of the interviewed is aware of having an intermediate level of knowledge, since they self-graded themselves with a 3 out of 5, 5 being having all the necessary knowledge about the topic of parasitism and 1 having none at all. $28 \%$ gave themselves 2 out of 5 , which makes it clear they are aware of their own lack of information on the topic.

Inhabitants of the community are encouraged to promote measures as simple as proper hand washing to improve the health and life of everyone.

Keywords: Parasitism; knowledge; behavior; practice; health determinants.

respiratorias altas y las dermatitis. También, es una de las más importantes causas de malnutriciones en poblaciones pediátricas. El diagnóstico se lleva a cabo por medio de estudios de laboratorio de heces fecales. El tratamiento tiende a ser muy efectivo en erradicar la patología una vez administrado de manera adecuada.

El objetivo de este estudio es determinar el nivel de información que posee la población de Paya sobre este tema, para así poder desarrollar mejores estrategias para su manejo en situaciones futuras.

Para ello, se propuso evaluar los conocimientos, actitudes y prácticas acerca de parasitosis intestinal en adultos que asistieron a consulta de atención primaria en la comunidad de Paya, municipio Baní, provincia Peravia, República Dominicana.

\section{Material y métodos}

Este estudio fue, por una parte, de carácter prospectivo ya que se extrajo la información utilizada durante un periodo establecido de tiempo. Por la otra, descriptivo, debido a que se expone de manera 
minuciosa la relación entre los conocimientos, actitudes y prácticas sobre parasitosis intestinal. Asimismo, es de corte transversal debido que se estudió la misma población durante un periodo determinado.

Para adquirir la información discutida se empleó un protocolo (ver sección de anexos) basado en una investigación previa, la cual fue adaptada por los autores.

En dicho protocolo, la información está dividida en tres secciones para evaluar los conocimientos, actitudes, y prácticas de la muestra acerca de la parasitosis intestinal.

Se tomaron en cuenta las fichas familiares de los pacientes, en la Unidad de Atención Primaria (UNAP), para tener un estimado de la población y aplicar los criterios de inclusión y exclusión de la muestra.

La población estudiada se trató de todos los pacientes de Paya Arriba que asistieron a la consulta en la Unidad de Atención Primaria de Paya. Siendo el universo todos los habitantes de la misma comunidad y una población total de 910 familias, con una muestra de 270 , representando el $30 \%$ de la población, con un nivel de confianza de $95 \%$ y un margen de error de $5 \%$ según la fórmula de cálculo de muestra.

Como criterio de inclusión se requirió que la persona fuese mayor de edad, y que se identificase a sí misma como cabecera del hogar, residente permanente de la comunidad de Paya Arriba y que de manera voluntaria participara en el estudio. Las casas se eligieron de forma aleatoria, distribuidas dentro de los límites de la comunidad.

Los criterios de exclusión constaron de lo siguiente: que fuese menor de edad, que no fuese residente permanente de Paya Arriba, que no se consultara previamente en la UNAP o que no accediera a participar en la investigación.

No se expuso la identidad del paciente para respetar el derecho de privacidad y exclusividad, y por tanto su integridad de ciudadano.

Como técnica de tabulación y procesamiento de datos se utilizó Microsoft Excel 2013.

\section{Resultados y Discusiones}

Gráfica 1. Rango etario de la muestra estudiada, mayo-julio 2018. $\mathrm{N}=270$

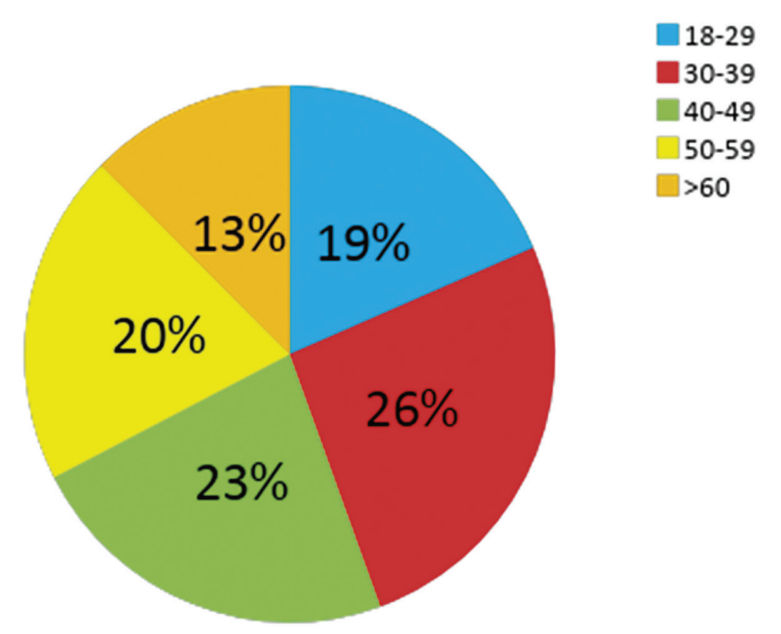

Fuente: elaboración propia. Internado Medicina Social, mayo-julio 2018.

Los 270 entrevistados se dividieron según edades, con base en rangos, iniciando por el criterio de inclusión: mayores de 18. El rango de 30 a 39 años resultó el más común, con un $26 \%$ del total de los entrevistados. Seguido por el grupo etario de 30 a 49 años, con un $23 \%$. A su vez, el grupo de 50 a 59 años con un $20 \%$. Luego, el grupo de 18 a 29 ańos con un $18 \%$. Para finalizar, el grupo etario de mayores de 60 ańos, con el menor de los porcentajes, $13 \%$. 
Gráfica 2. Género de la muestra entrevistada, mayo-julio 2018. $\mathrm{N}=270$

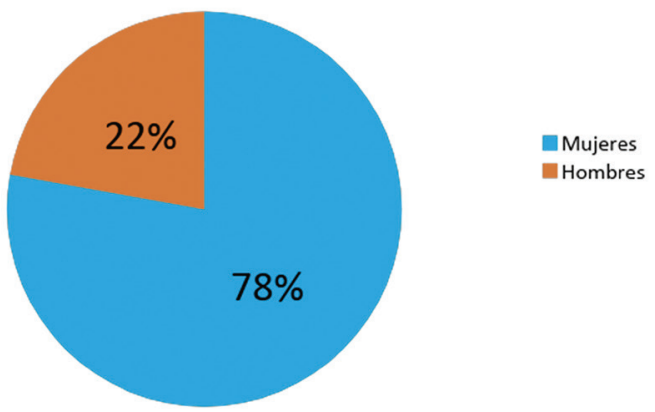

Fuente: elaboración propia. Internado Medicina Social, mayo-julio 2018.

Este gráfico evidencia la cantidad de hombres entrevistados, representando el $22 \%$ de la muestra; a su vez, la cantidad de mujeres entrevistadas que representan el $78 \%$ de la muestra, es decir, la mayoría.

Gráfica 3. Número de habitantes por casa, mayo-julio 2018. $\mathrm{N}=270$

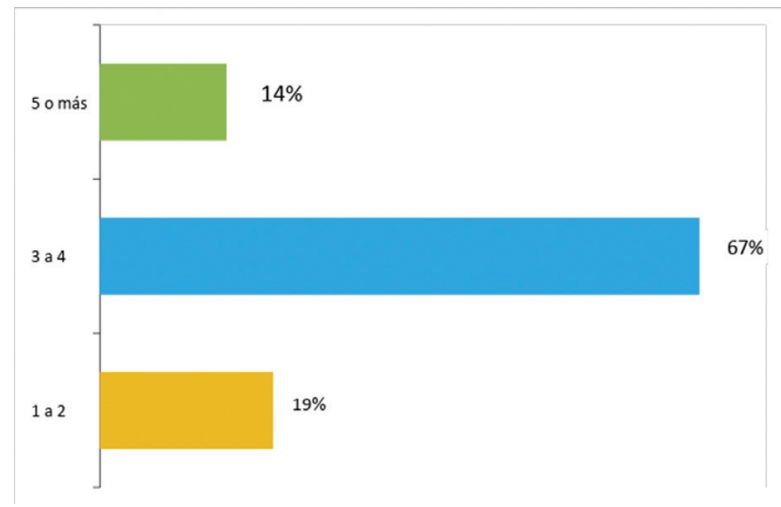

Fuente: elaboración propia. Internado Medicina Social, mayo-julio 2018.

Esta gráfica representa la cantidad de habitantes por casa, siendo el $67 \%$ el porcentaje más significativo, que dice que por cada casa habían alrededor de 3 a 4 personas, seguido de un $19 \%$ en viviendas con 1 a 2 personas y un $14 \%$ obtenido por las viviendas con cinco o más personas.

72 | Ciencia y Salud 2019; 3(3): 69-78 • Casos clínicos
Gráfica 4. Nivel de educación de la muestra a estudiar, mayo-julio 2018. $\mathrm{N}=270$

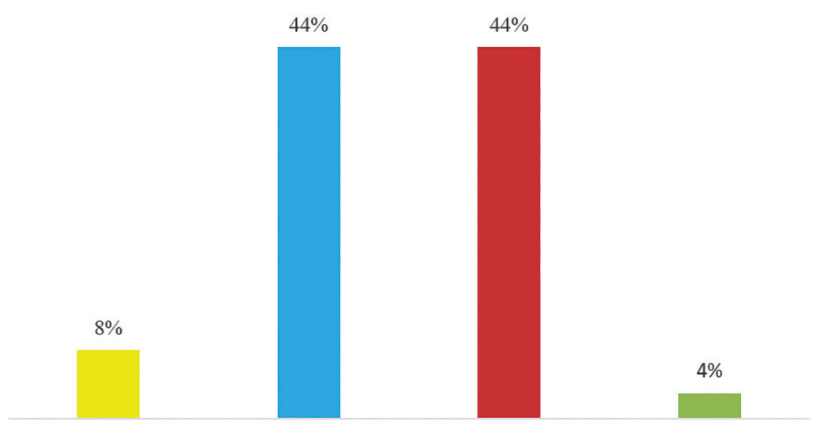

Fuente: elaboración propia. Internado Medicina Social, mayo-julio 2018.

Esta gráfica muestra el nivel de escolaridad de los entrevistados, coincidiendo el nivel de básica y bachiller, con el mayor de los porcentajes, los cuales representan el $44 \%$, respectivamente. Para un $8 \%$ de encuestados que llegaron hasta nivel inicial y $4 \%$ de universitarios, siendo este el más bajo.

Gráfica 5. Conocimiento de la población sobre qué es un parásito, Mayo-julio 2018. N=270

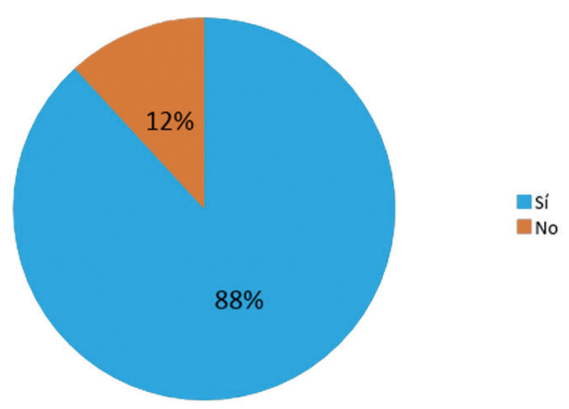

Fuente: elaboración propia. Internado Medicina' Social, mayo-julio 2018.

Esta gráfica muestra los conocimientos que creen tener los entrevistados sobre si saben o no lo que es un parásito. Representando el mayor porcentaje un $88 \%$ de personas que sí creen saber lo que es un parásito, y una minoría, del $12 \%$, que respondió no saber lo que son los parásitos. 
Gráfica 6. Percepción de qué es un parásito por parte de la población, mayo-julio 2018

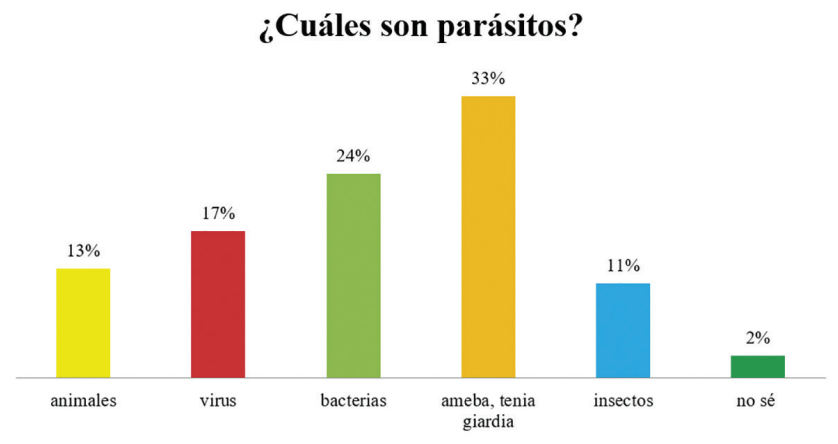

Fuente: elaboración propia. Internado Medicina Social, mayo-julio 2018.

Esta gráfica permite ver la percepción que tienen los entrevistados acerca de cuáles opciones para ellos representan un parásito. Acertando la mayoría en que corresponde a Ameba, Giardia y Tenia con un $33 \%$. Mientras que un $24 \%$ de la población aseguró los parásitos son bacterias; $17 \%$ dijo que son virus; $13 \%$ asegura que son animales; $11 \%$ insectos y un $2 \%$ respondió que no sabían lo que eran.

Gráfica 7. Cantidad de entrevistados que asegura haber visto parásitos, mayo-julio 2018. (N=270)

\section{¿Ha visto un parásito?}

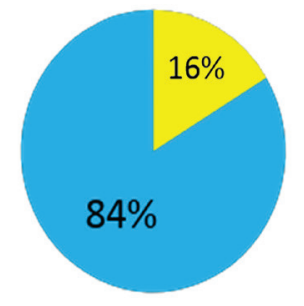

Fuente: elaboración propia. Internado Medicina Social, mayo-julio 2018.

La gráfica representa el porcentaje de entrevistados que dijo haber visto o no un parásito alguna vez. Solo un $16 \%$ aseguró haberlos visto, y un $84 \%$ asegura que nunca ha visto un parásito.
Gráfica 8. Incidencia de parasitosis en los entrevistados, mayo-julio 2018. ( $\mathrm{N}=270)$.

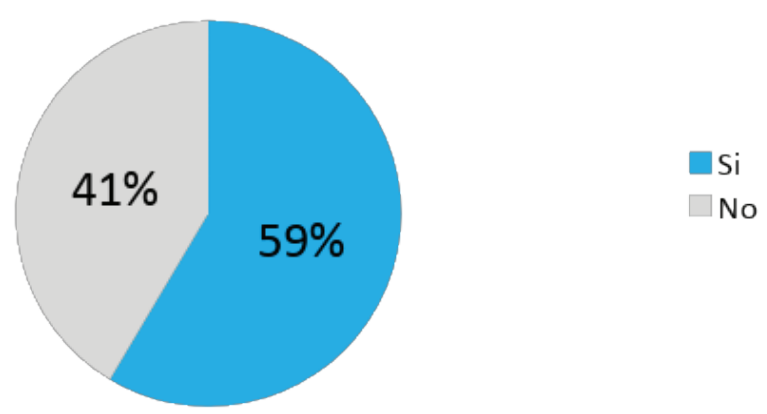

Fuente: elaboración propia. Internado Medicina Social, mayo-julio 2018.

La gráfica representa la cantidad de personas que alguna vez padeció por alguna parasitosis, con un $59 \%$ de casos donde la muestra tomada es de $\mathrm{N}=270$, siendo 158 casos previos de parasitosis y 112 casos, que representan el $41 \%$, de personas que no han padecido parasitosis nunca.

Gráfica 9. Cantidad de casos de parasitosis por vivienda, mayo-julio 2018

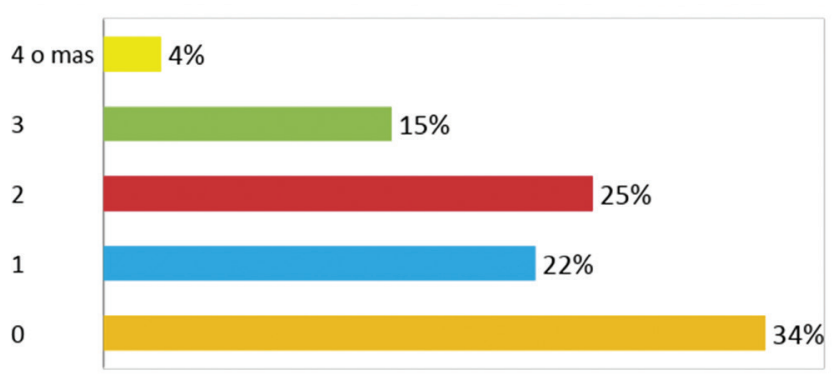

Fuente: elaboración propia. Internado Medicina Social, mayo-julio 2018.

En esta gráfica se muestran cuántas personas, por cantidad de habitantes, por vivienda, padecieron parasitosis. El mayor porcentaje, $34 \%$, representa que nadie padeció de parasitosis en esa vivienda. Mientras que el $25 \%$ de los entrevistados aseguró que al menos dos personas en esa vivienda han padecido de parasitosis. Un $22 \%$ de los entrevistados asegura que en la vivienda solo una persona padeció 
de parasitosis; $15 \%$ dijo que por lo menos tres personas habían padecido parasitosis y la minoría, $4 \%$, dijo que cuatro o más personas en la vivienda habían padecido parasitosis alguna vez.

Gráfica 10. Síntomas y signos que presentaron los entrevistados y que les hicieron pensar que padecían parasitosis, mayo-julio 2018

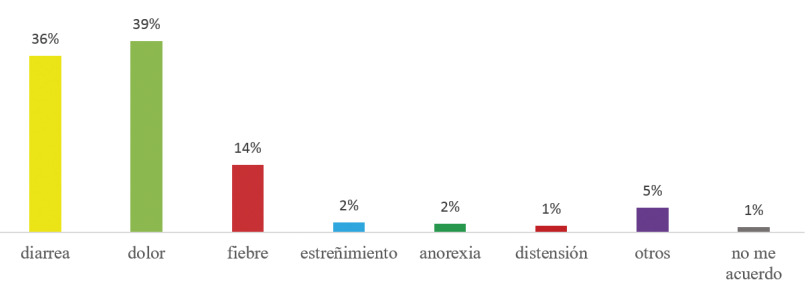

Fuente: elaboración propia. Internado Medicina Social, mayo-julio 2018.

Esta gráfica nos indica los síntomas y signos que los entrevistados manifestaron, o qué le hacen pensar que podrían tener una parasitosis. Representando el dolor y la diarrea los síntomas más comunes para un $39 \%$ y un $36 \%$ respectivamente. Por otro lado, $14 \%$ aseguró tener fiebre; $2 \%$, estreñimiento; $2 \%$, anorexia; $1 \%$ presentó distención abdominal; $5 \%$ presentó otros, como vómito, náuseas, ictericia y flatulencia. Finalmente, $1 \%$ de los entrevistados no recuerda.

Tabla 1. Percepción de los entrevistados acerca de cómo adquirir parásitos, mayo-julio 2018

\begin{tabular}{|l|c|c|}
\hline $\begin{array}{l}\text { ¿Dónde cree que se pueden adquirir } \\
\text { los parásitos? }\end{array}$ & Repuestas & Porcentaje \\
\hline Dar la mano a otra persona & 46 & $15 \%$ \\
\hline Por la saliva & 66 & $22 \%$ \\
\hline Por moscas & 28 & $9 \%$ \\
\hline Tener animales domésticos & 30 & $10 \%$ \\
\hline Por el agua de consumo & 18 & $6 \%$ \\
\hline Por las verduras y frutas & 18 & $6 \%$ \\
\hline Por estar descalzo & 26 & $9 \%$ \\
\hline Por mala higiene & 52 & $17 \%$ \\
\hline Por no lavarse las manos & 10 & $3 \%$ \\
\hline No sé & 8 & $3 \%$ \\
\hline Total & 302 & $100 \%$ \\
\hline
\end{tabular}

Fuente: elaboración propia. Internado Medicina Social, mayo-julio 2018.
Esta tabla muestra dónde creen los entrevistados que se pueden adquirir los parásitos. El porcentaje más alto lo obtuvo la idea de que a través de la saliva se pueden adquirir, con un $22 \%$ del total de las opciones respondidas, mientras que el $3 \%$ de los entrevistados asegura que se adquieren por no lavarse las manos, lo cual es una cifra alarmante.

Tabla 2. Percepción de los entrevistados acerca de dónde consideran que se alojan los parásitos, mayo-julio 2018

\begin{tabular}{|c|c|c|}
\hline $\begin{array}{c}\text { ¿Dónde cree que } \\
\text { alojan los parásitos? }\end{array}$ & Respuestas & Porcentaje \\
\hline Piel & 62 & $11 \%$ \\
\hline Corazón & 12 & $2 \%$ \\
\hline Cerebro & 58 & $11 \%$ \\
\hline Hígado & 38 & $7 \%$ \\
\hline Estómago & 192 & $36 \%$ \\
\hline Intestinos & 166 & $31 \%$ \\
\hline Rińones & 9 & $2 \%$ \\
\hline Total & 537 & $100 \%$ \\
\hline
\end{tabular}

Fuente: elaboración propia. Internado Medicina Social, mayo-julio 2018.

En esta tabla se muestran los órganos en donde podrían alojarse los parásitos de acuerdo con lo respondido por los entrevistados. Un $36 \%$ de ellos (192 entrevistados) afirma que se alojan en el estómago, siendo la cifra más alta. Y una minoría de $2 \%$ asegura que pueden hospedarse en los riñones y en el corazón, respectivamente. 
Gráfica 12. Cantidad de veces al día que los entrevistados afirman lavarse las manos, mayo-julio 2018. $\mathrm{N}=270$

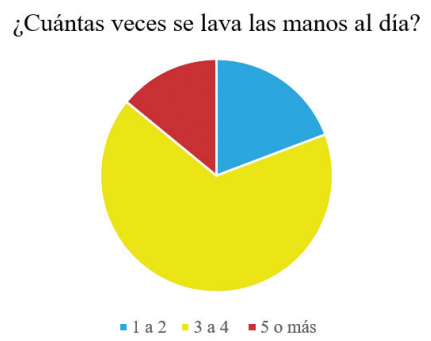

Fuente: elaboración propia. Internado Medicina Social, mayo-julio 2018.

La gráfica 12 representa la cantidad de veces que los entrevistados afirman lavarse las manos en un día. $76 \%$ de ellos dicen lavarse las manos cinco o más veces al día, mientras que $24 \%$ de los entrevistados manifestó que se lavan las manos de tres a cuatro veces al día. Nadie dijo lavarla menos de tres veces al día.

Gráfica 13. Prácticas con relación a la parasitosis por parte de los entrevistados, mayo-julio 2018. N = 270

¿Qué hace cuando cree que tiene parásitos?

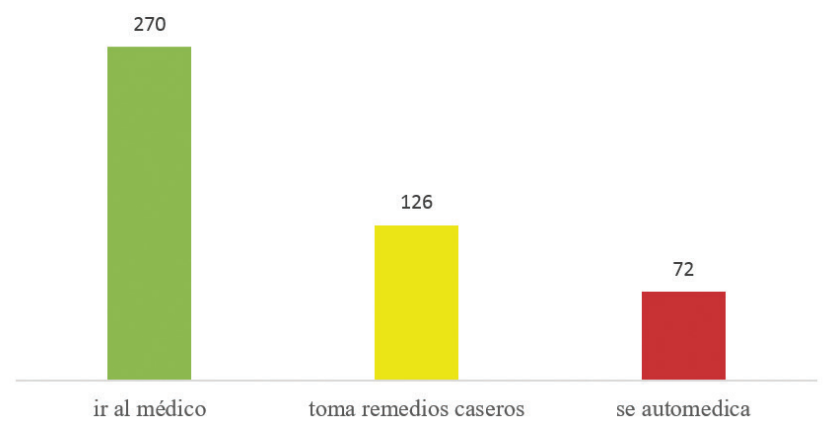

Fuente: elaboración propia. Internado Medicina Social, mayo-julio 2018.

La gráfica 13 representa las prácticas por parte de los entrevistados cuando creen tener una parasitosis. Con una muestra de $\mathrm{N}=270$; donde todos afirman buscar ayuda médica; 126 de los entrevistados preparan y/o toman remedios caseros y 72 de ellos se automedican.
Tabla 3. Actitudes y prácticas de los entrevistados luego de ir al médico, mayo-julio 2018. N = 270

\begin{tabular}{|l|c|c|c|}
\hline De haber ido al médico ... & Sí & No & $\begin{array}{c}\text { No Recuer- } \\
\text { do }\end{array}$ \\
\hline ¿Se le indicó un coprológico? & $69 \%$ & $10 \%$ & $21 \%$ \\
\hline ¿Se lo realizó? & $69 \%$ & $10 \%$ & $21 \%$ \\
\hline ¿Recibió medicación? & $69 \%$ & $10 \%$ & $21 \%$ \\
\hline ¿Completó el medicamento? & $50 \%$ & $22 \%$ & $28 \%$ \\
\hline $\begin{array}{l}\text { Al finalizar el tratamiento, ¿se } \\
\text { repitió el coprológico? }\end{array}$ & $50 \%$ & $30 \%$ & $20 \%$ \\
\hline
\end{tabular}

Fuente: elaboración propia. Internado Medicina Social, mayo-julio 2018.

Esta tabla representa las actitudes y prácticas de los entrevistados luego de haber visitado un médico; $69 \%$ (186 casos) de ellos asegura que se les indicó un coprológico; $10 \%$ (28 casos) asegura que no se les indicó un coprológico y $21 \%$ (56 casos) dijo no recordar si se lo indicaron o no. Esta misma cantidad de porcentajes y de casos es igual para las personas que se realizaron el coprológico o no luego de que se les fue indicado, o que no recuerda; y las mismas cantidades para las personas que dicen haber recibido o no medicación y las que no lo recuerdan.

Por otro lado, $50 \%$ (136 casos) aseguran haber completado el esquema de medicamentos; $22 \%$ (60 casos) no completaron el esquema de medicamentos tal y como les fue indicado y $28 \%$ (74 casos) no recuerda si completó o no el esquema de medicación.

Finalmente, $50 \%$ (126 casos) de los entrevistados asegura que se les volvió a repetir el coprológico luego de haber finalizado el tratamiento; $30 \%$ (80 casos) no se realizó un coprológico nuevamente, luego de finalizado el tratamiento, y $20 \%$ (64 casos) no recuerda si se repitió o no el coprológico luego de terminar el tratamiento. 
Gráfica 14. Parentesco de la persona a cargo cuando el entrevistado se enferma, mayo-julio 2018.

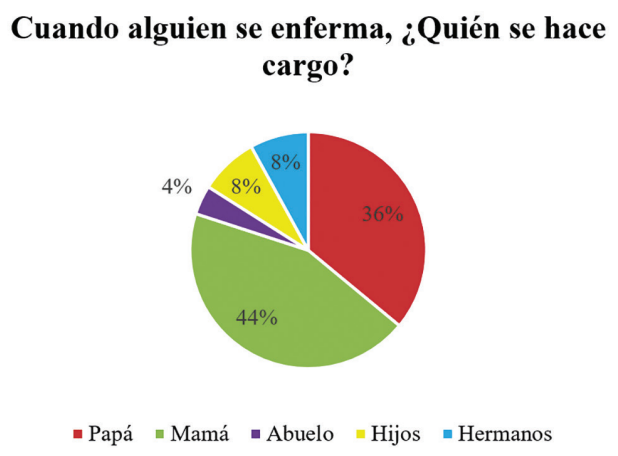

Fuente: elaboración propia. Internado Medicina Social, mayo-julio 2018.

Esta gráfica representa quién se hace cargo en la vivienda cuando algún miembro del hogar se enferma. Siendo el mayor porcentaje para las madres, con un $44 \%$, seguido de un $36 \%$ representado por los padres, y un $8 \%$ para hijos y hermanos respectivamente, en el caso de los adultos mayores; $4 \%$, la minoría, representada por los abuelos en el caso de adultos jóvenes que aún dependen económicamente de terceros.

Gráfica 15. Frecuencia de recogida de basura en casa de los entrevistados, mayo-julio 2018.

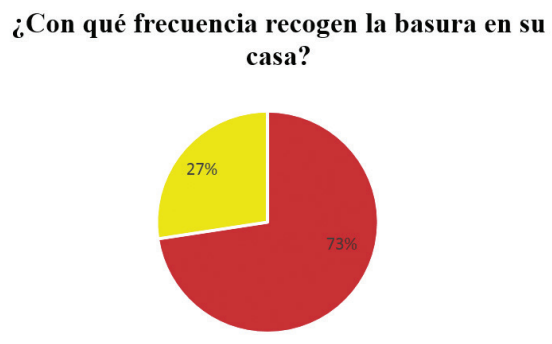

- Una vez a la semana = Dos veces a la semana

Fuente: elaboración propia. Internado Medicina Social, mayo-julio 2018.

Esta gráfica muestra la cantidad de veces por semana que los entrevistados se deshacen de la basura y los desechos en sus viviendas. El 78 \% (porcentaje mayor) saca la basura una vez por semana. El
$27 \%$ restante la saca hasta dos veces por semana. Ninguno de los entrevistados la saca más de dos veces por semana, según respondieron, ni menos de una vez por semana.

Gráfica 16. Cantidad de entrevistados que asegura haber recibido charlas educativas acerca de la parasitosis en algún momento previo, mayo-julio 2018

¿Ha recibido charlas educativas?

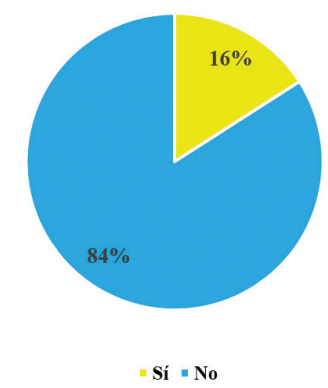

Fuente: elaboración propia. Internado Medicina Social, mayo-julio 2018.

Esta gráfica muestra el porcentaje de los entrevistados que asegura haber recibido una charla educativa previa acerca de parasitosis, lo cual representa el $16 \%$ de los entrevistados. El $84 \%$ restante dijo nunca haber recibido una charla educativa sobre este tema.

Gráfica 17. Autoevaluación de los entrevistados acerca de los conocimientos que creen tener acerca de la parasitosis, mayo-julio 2018

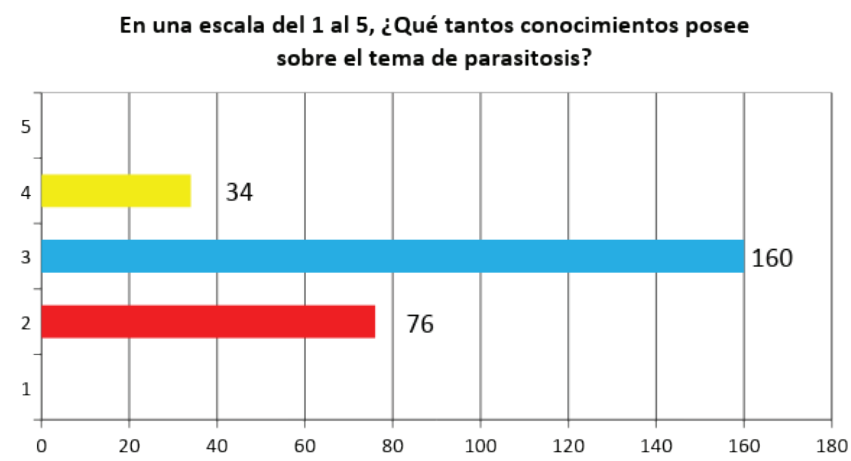

Fuente: elaboración propia. Internado Medicina Social, mayo-julio 2018. 
La gráfica 17 es más bien una escala de autoevaluación de los entrevistados; ellos mismos dan una calificación a la cantidad de conocimientos acerca de parasitosis que creen tener. Donde 160 de los casos, con una muestra de $\mathrm{N}=270$, se autocalificó con un 3 de 5 , siendo el 5 el valor más alto de la calificación, y el 1 el más bajo. Por su parte, 76 de los entrevistados se autocalificó con un 2 de 5 puntos $y$, finalmente, 34 personas se autocalificaron con un 4 de 5 puntos.

\section{Conclusiones}

Una vez realizada esta investigación queda demostrada que la población a estudiar posee un nivel de conocimientos con relación al tema de parasitosis intestinal que, si bien algunos están acertados, en su mayoría son básicos o errados.

Según los datos obtenidos la mayor parte de la población estudiada, la cual representó el $26 \%$, se encuentra en la cuarta década de la vida, seguido de un $23 \%$ representado por las personas en la quinta, y que bien pueden ser agentes promotores de cambio, tener los conocimientos adecuados, modificar las actitudes y perfeccionar las prácticas de la población, para poder prevenir en lo posible cualquier tipo de parasitosis por simple que parezca, en lugar de curarlas.

Los datos obtenidos arrojan una alarmante suma de personas de la población que, de forma equívoca, afirman que el hecho de no lavarse las manos no influye en adquirir parásitos; lo cual pone de manifiesto la importancia de recalcar en los habitantes lo imprescindible de acciones tan sencillas como el lavado de manos para promover la salud y la vida.

A pesar de que un $76 \%$ de la población afirma lavarse las manos cinco o más veces al día, otra gráfica demuestra algo muy contrastante, ya que un importante porcentaje de los entrevistados aseguró que al menos dos personas en la misma vivienda habían padecido parasitosis; y un considerable porcentaje afirmó que de tres a cuatro personas o más, también padecieron parasitosis alguna vez en la misma vivienda, incluso, de manera simultánea, por medio del contagio.

La mayor cantidad de entrevistados que aseguró haber visto parásitos era directamente proporcional a la edad, los de edad más avanzada refirieron haber visto parásitos, específicamente lombrices, lo cual no es común hoy en día. Cabe destacar que la muestra estudiada tiene un nivel de conocimiento aceptable acerca de dónde y cómo creen que pueden contraer parásitos. En ese mismo orden, todos los entrevistados piensan que podrían llegar a morir por una parasitosis, lo cual es un factor positivo, como medio para incentivarles a prevenir las mismas.

Todos los entrevistados aseguraron utilizar agua de la llave y agua de botellones para consumo doméstico. Esto sumado a que el $100 \%$ de los entrevistados tiene un bańo privado en la vivienda y que la recogida de basura oscila entre una y dos veces por semana, factores sumamente positivos, que encaminan a la población a mayores estándares de calidad de vida.

Según los datos arrojados por la investigación, se puede decir que la población estudiada tuvo buen seguimiento clínico, cumpliendo con los protocolos de coprológico-medicamento-coprológico. El 60 \% de los entrevistados está consciente de que tiene un nivel intermedio de conocimientos ya que se autoevaluaron a sí mismos con un tres de cinco, siendo el cinco el mayor puntaje. Mientras que un $28 \%$ se autoevaluó con dos de cinco, lo que pone de manifiesto que está consciente de la necesidad de educación en el tema. 


\section{Bibliografía}

1. WHO. Report of the third global meeting of the partners for parasite control. Geneva: Strategy Development and Monitoring for Parasitic Diseases and Vector Control, Communicable Diseases Control, Prevention and Eradication, Communicable Diseases; 2005.

2. PAHO. First session of the subcommittee on program, budget, and administration of the executive committee. Washington: Pan American Health Organization world health organization; 2007.
3. Información Farmacéutica de la Comarca (INFAC). Parasitosis Intestinal. Boletín INFAC. 2009;17(2) Disponible en http://www.sld.cu/ galerias/pdf/sitios/apua-cuba/parasitosis_intestinales.pdf 